\title{
Modern Electronic Warfare Signal Processing Based on Analog-to-Information Conversion
}

\author{
Wen-Zhe Wang ${ }^{1, a}$, Hua Wu ${ }^{1, b}$ and Ji-Yuan $\mathrm{He}^{1, \mathrm{c}}$ \\ ${ }^{1}$ Aeronautics and Astronautics Engineering College, Air Force Engineering University, Xi' an \\ 710038, China.
}

\author{
a524940802@qq.com, b15129291728@163.com, 'wangwenzhe9008902@gmail.com
}

Keywords: Compressed sensing, measuring matrix, parallel method, low-speed ADCs sampling.

\begin{abstract}
Many problems in radar signal processing involve radio frequency (RF) signals of very high bandwidth. This presents a serious challenge to systems that might attempt to sample these signals by the Shannon/Nyquist sampling theorem. However, the information level of the signal is often far lower than the actual bandwidth, while the novel theory of compressive sensing enables the direct analog-to-information converter (AIC) of compressible signals at sub-Nyquist acquisition rates. In this paper we propose a system that uses a parallel bank of low-rate analog-to-digital converter (ADC) to produce a low-rate set of digital measurements. Our AIC is inspired by the recent theory of Compressive Sensing (CS). We generalize the CS theory to fit it into the continuous-time sparse signal backdrop, explain AIC system in the CS context, and discuss practical issues regarding implementation. Simulation shows that the architecture is particularly apropos for wideband signals that are sparse or nearly sparse in the time-frequency domain.
\end{abstract}

\section{Introduction}

The simpleness, stability, and low cost of digital signal processing (DSP) have popularized the ADC-based on the Nyquist sampling theorem at the front-end of radar systems. Along with the development of modern radar technology, the radar signal system is becoming more and more complicated, enhancing the pulse density of electronic environment from 1 million to 10 million, or more per minute. The ever growing demand for data prompts the use of high-bandwidth signals, on which the samping rates dictated by the Shannon-Nyquist theorem impose severe challenges. This makes the applications like radar detection and sampling systems in general greatly exceeds current capabilities for pushing the performance of ADCs toward their physical limits. It could be decades before ADCs based on current technology become fast and precise enough for these applications and avoid the deluge of data swamping back-end DSP algorithms. However in application, only a tiny fraction of gained information is actually relevant, the wideband signals in many RF applications often have a large bandwidth but a relatively small "information rate".

CS is a promising field that has attracted considerable research interest in the signal processing community since its introduction only several years ago [1,2], which exploits this knowledge to achieve signal acquisition using fewer measurements than those prescribed by the Nyquist theorem for the signals are compressible in some transform domain (such as Fourier, wavelet, etc.) [3, 4]. By leveraging the CS theory, an AIC was designed to acquire samples at a lower rate while successfully recovering the compressible signal of interest. Through measuring the key information, CS can reconstruct the non-cooperative original signal with high speed. Thus, the low speed ADCs can complete the task of acquiring signal of large-bandwidth and implement signals with large dynamic range by parallel method. The CS algorithm, establishes mathematically that a relatively small number of non-adaptive, linear measurements can acquire all of the information necessary to faithfully reconstruct sparse or compressible signals. An intriguing aspect of the theory is the central role played by randomization. 


\section{Compressible analysis}

The working frequency range of radars is mainly around $2 \mathrm{GHz} \sim 18 \mathrm{GHz}$. The number of signals acquired from the radar receiver is single in high probability, whereas the probability of more than one arriving signals is much lower. Even the occasion of multi-signals appeared, the number of signals is finite. Furthermore, in limited time, the bandwidth can be achieved by pulse-modulated signal is limited, thus it can be viewed as multiple radio narrowband signals in the range of broadband. Thus, the Fourier coefficients of the received signal are nearly sparse. The sparsity makes the application of CS highly probable. In a word, CS can be employed in the all-band radar receiver of its frequency or space domain.

\section{AIC system design}

CS framework. Since the CS receiver is directly sensing the compressive analog signal, when the antenna incepts electromagnetic signal, it is first transformed in transform domain, i.e. non-adaptive mapping of no-loss compressed measurement.

Under the most circumstances of radar application, the processed signals is multi-band signals containing many components of interest, and its co-support of Fourier transform is $\mathrm{m}$ non-crossing frequency ranges (the position of every frequency range is random and uncharted). Aimed at multiband signals, we designed signal processing framework based on parallel multi-channel OMP iterative reconstruction as Fig 1. In this framework, there is no need to know the information of the carrier frequency, and the signal bandwidth is unimportant. Hence, the technique of AIC parallel acquisition is suitable for the signal sampling system of the receiver.

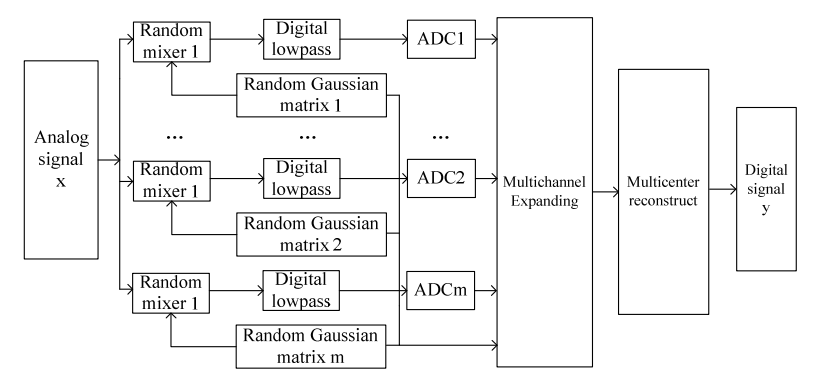

Fig. 1 Signal processing chart

Multi-band analog signal $x$ enters $m$ channels simultaneously as shown above. The analog signal enter the mixer of channels for mixing first, and the mixing functions of each channel are i.i.d random periodic signals but put up different waveforms. Then they pass though the digital low pass filters and ADCs respectively to implement the low speed sampling and gain signal y from AIC. Meanwhile, the purpose of mixers is to carry the signal to basic band and the digital low pass filters aim to wipe off the high frequency components.

Program design. The big difference between AIC and traditional digital processing is the acquisition of the electromagnetic signals, and the following processing is basically the same, such as parameter estimation, feature extraction, signal detection, performance evaluation. The fact makes AIC compatible to traditional computer in the display and control aspect. It is apparent that the prominent characteristics of AIC are compression and reconstruction in signal processing.

The basic thoughts of CS receiver lies in multiple channels parallel compressive reconstruction: the non-adaptive compressive measurement of acquired signal is essential for the lossless processing; Thus the precise reconstruction of the whole information from the sparse domain in the compressed sampling signals can hardly be achieved, however, the original signal can still be reconstructed in 
high probability by optimization algorithms, paving the way for the following processes, such as parameter estimation.

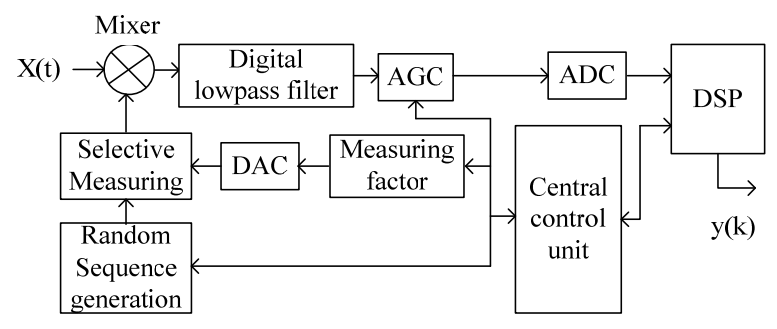

Fig. 2 Signal processing design

Signal processing design mainly includes two parts, i.e. the analog front-end module which includes the mixers and digital low-pass filters, along with the data collection and reconstruction modules. Considering AIC sampling primarily relies on the condition of small mutual coherence, this article adopts Gaussian random matrix and Fourier basis as sensing matrices.

\section{Simulation}

Based on the theory and design above, we stimulate it via MATLAB R2014a of Window XP station. For more intuitionistic, regarding the compounding of sine signals as original signal. Based on the great incoherent of random matrix and for more convenient in selecting effective information, measuring matrix adopts $\mathrm{M} \times \mathrm{N}=64 \times 256$ normal distribution random matrix. Reconstructing algorithms is OMP multiple iterate algorithm.
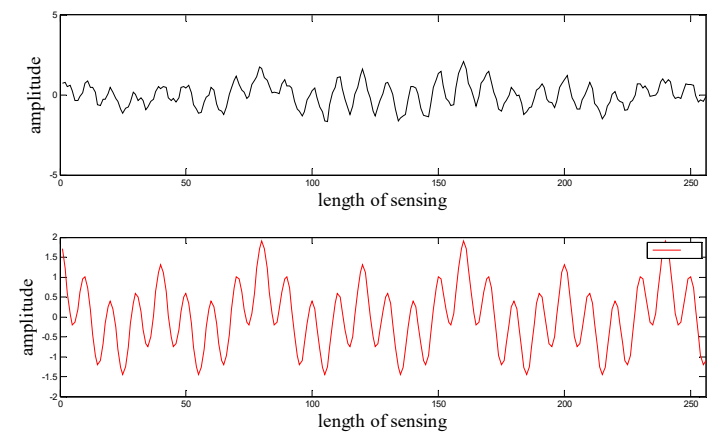

(a)

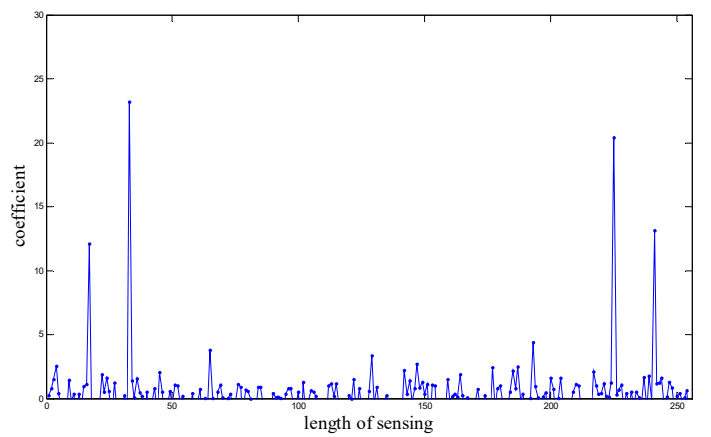

(b)

Fig. 3 (a) Comparisons of reconstructed signal employing AIC vs. original (with noise). The difference between the reconstruction and the original signal is hardly noticeable in CS recovery. As we described in under sampling and Sparse Signal Recovery, the signal can be perfectly recovered from just 20 incoherent measurements. (b) Project location and coefficients of reconstructed signal. Only few coefficients are large while other approximate to zero and the few is interested.

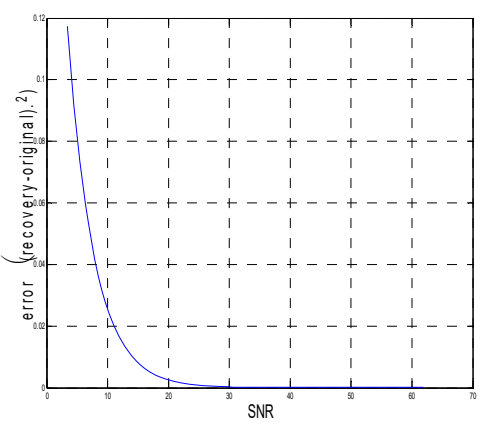

(a)

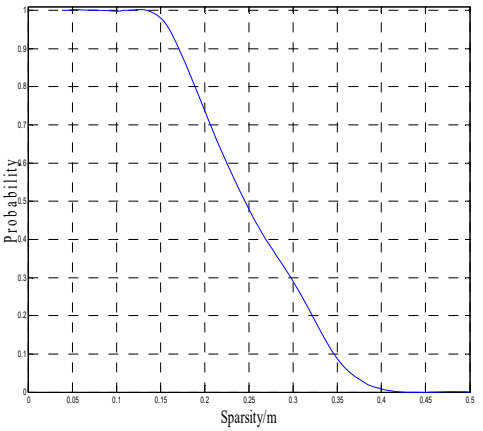

(b)

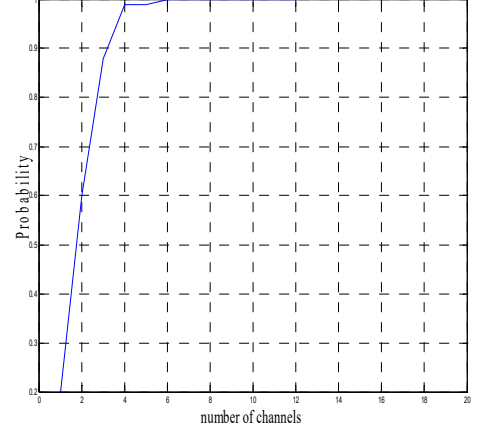

(c)

Fig. 4 (a) CS recovery error. (b) Probability of different sparse (without projection). (c) Probability of different number of channels. The reconstruction probability of different parameter includes SNR, 
sparse and channel number. The experiment shows that the radar receiver will work better when the SNR is $20 \mathrm{dBw}$, sparse $\mathrm{m}<0.15$ and number of channel is equal or more than 4 .

Table 1 The CPU time of different algorithm

\begin{tabular}{cccc}
\hline Time & Nyquist & CS & $\begin{array}{c}\text { Relatively } \\
\text { ((Nyquist-CS)/Nyquist) }\end{array}$ \\
\hline Total & $0.0878 \mathrm{~s}$ & $0.0685 \mathrm{~s}$ & $21.98 \%$ \\
Front-end & $0.0471 \mathrm{~s}$ & $0.0219 \mathrm{~s}$ & $53.50 \%$ \\
\hline
\end{tabular}

Checking the complexity of the two kinds of operations by calculating the running-time of sampling methods, it can be noted that the totally cost time of AIC process decreased $21.98 \%$ relatively vs. Nyquist. Moreover, the front-end of AIC takes only account for $20.18 \%$ of the total time, and of Nyquist, it takes its 53.64\%. So the AIC time-consuming of the front-end is much less for transferring the computational complexity of the reconstructed signal to back-end i.e. computer central control unit significantly reducing the work intensity of the radar receiver's front-end and increasing its flexibility and instantaneity.

\section{Summary}

This paper presents the design and implementation of an AIC capable of compressively sensing modes of operation on the electronic warfare radar receiver. By integrating parallel method with an efficient reconstruction algorithm, the design has lower complexity compared to conventional Nyquist rate sampling architectures, while at the same time increasing the power efficiency of the sampling operation for signals with sparse frequency support. For this, parallel processing and compressive measuring gain higher instantaneous bandwidth and dynamic range without bandwidth limit of ADCs, completely intercepting the frequency range of multiple radar pulses reached at the same time and measuring parameter. Measured results of the AIC demonstrate its ability to acquire signals with sparse frequency support. This is a critical improvement compared to the Nyquist rate, which is already a significant limit to the state of the art radar implementations. In our future realization, one could incorporate the proposed AIC within the architecture proposed for sparse band-limited signals due to its simplicity, feasibility and flexibility in handling changes in frequency bands. In this context the group sparsity penalized algorithm is proposed in this paper and shown to have better performance than 11-minimization for recovering the occupied bands when the number of occupied bands is sparse. This provides a novel solution ideology to the task.

\section{References}

[1] Marco F. Duarte, Yonina C. Eldar, Structured compressed sensing: From theory to applications, IEEE Transactions on signal processing. 59(9), pp.4053-4085, 2011.

[2] Doris Bao, Pasquale Daponte, Luca De Vito, Sergio Rapuano, Frequency-domain characterization of random demodulation analog-to-information converters, ACTA IMEKO. 4(1), pp.111- 120, 2015.

[3] Michael Trakimas, Robert D., Angelo, Shuchin Aeron, et al., A compressed sensing analog-to-information converter with edge-triggered sar adc core, IEEE Transactions on circuits and systems. 60(5), pp.1135-1148, 2013.

[4] Storm K., Murthy V., Selesnick I., Pillai U., Sparsity-based methods for interrupted radar data reconstruction, Radar Conference 2012 IEEE. pp.0107-0111, 2012. 